\title{
Stereotactic Radiosurgery of Colorectal Carcinoma Brain Metastasis: A Multi- Institutional Experience.
}

Aizik L. Wolf ${ }^{1}$, Sachin Batra ${ }^{2}$, Pietro Bortoletto ${ }^{3}$, Chetan Bettegowda ${ }^{4}$, Beatriz E. Amendola 5 , Laurie Blach ${ }^{6}$, Lawrence Kleinberg ${ }^{7}$, Daniele Rigamonti ${ }^{8}$

1. Miami Neuroscience Center, Larkin Community Hospital 2. Department of Neurosurgery, Johns Hopkins Hospital 3. Feinberg School of Medicine 4. Department of Neurosurgery, The Johns Hopkins University School of Medicine, Baltimore Maryland 5. Innovative Cancer Institute 6. Miami Neuroscience Center, Mount Sinai Medical Center, Miami Beach, Florida 7. Department of Radiation Oncology, Johns Hopkins University School of Medicine 8. Department of Neurosurgery, The Johns Hopkins University School of Medicine

$\square$ Corresponding author: Aizik L. Wolf, awolf@larkinhospital.com Disclosures can be found in Additional Information at the end of the article

\section{Abstract}

Purpose: Colorectal cancer is one of the most common cancers with one of the poorest survival rates following brain metastasis. Only a few studies have described outcomes after radiosurgery and predictors of mortality in these patients. We describe a multi-center experience in the treatment of colorectal cancer metastases to the brain using stereotactic radiosurgery.

Material and Methods: A retrospective review of 66 patients treated over 1993 to 2009 with at least one follow-up was undertaken. Median time to death and its predictors were calculated using Kaplan Meier survival analysis and Cox proportional hazard model.

Results: Overall median survival for patients with colorectal cancer was 4.05 months with a mortality of 58 (84.06\%) patients over a mean follow-up of 7.03+12.44 months. Both survival analysis and multivariate analysis revealed KPS $>70$ as the only significant predictor of survival (median survival KPS > 70: 6.91 months versus KPS < 70: 1.74 months, $\mathrm{p}=0.004$; Hazard ratio for KPS $>70$ was $0.31, \mathrm{p}=0.001$ ). When total volume did not exceed $5.4 \mathrm{cc}^{3}$, the patients with volume distributed over fewer lesions had improved survival. No such association was observed with higher volumes.

Conclusions: SRS moderately improves survival of patients with colorectal cancer metastatic to brain. Higher KPS predicts favorable prognosis in these patients.

\section{Published 05/17/2012}

\section{(๑) Copyright 2012}

Wolf et al. This is an open access article distributed under the terms of the Creative Commons Attribution License CC-BY 3.0., which permits unrestricted use, distribution, and reproduction in any medium, provided the original author and source are credited.
Categories: Radiation Oncology, General Surgery, Neurosurgery

Keywords: brain metastasis, colorectal cancer, stereotactic radiosurgery, Volume distribution

\section{Introduction}

Colorectal cancer is the fourth most common form of cancer constituting $9.4 \%$ of all cancers in men and $10.1 \%$ of cancers diagnosed in women worldwide [1]. Despite such frequent occurrence of colorectal cancer, metastatic lesions to the brain are rare and occur in only $0.64-6 \%$ of patients. In comparison, other common cancers like non-small cell lung cancer and breast cancer have rates of $50 \%$ and $12 \%$ developing brain metastasis, respectively [2-5]. Although 
lung and liver are the most frequent sites of metastases from colorectal carcinoma with increasing survival, distant lesions, such as the brain, are becoming more frequent [6-8].

Although surgical resection with postoperative radiotherapy has been the standard of care in the management of cerebral metastases [9-11], stereotactic radiosurgery provides an alternative treatment that spares the surrounding parenchyma, has less treatment related morbidity than surgery [12], and can access lesions that are inoperable. Radiosurgery in well-selected patients enhances survival from 2.1 - 3 months in those who receive palliative treatment using steroids or whole brain radiation therapy [13-15] to 7.1 - 11 months. Unfortunately, most existing studies are based on heterogeneous histological groups or pertain to the primaries most likely to metastasize to brain, like non-small cell lung carcinoma [16-18], breast carcinoma [19-21], melanoma [22] or renal cell carcinomas [23-24]. Only three studies, to our knowledge, have reported outcomes of radiosurgical treatment of brain metastasis from colorectal primaries - a condition that is becoming increasingly common [3, 25-26]. The challenge in grouping colorectal cancer patients with other tumor types that more commonly spread to the brain is the difficulty in accounting for extracranial disease burden. For example, colorectal adenocarcinoma frequently spreads to the liver and lungs, sites that are not as commonly seen in other tumor types. Therefore, systemic disease burden is often a confounding factor when studying patients with colorectal metastases to the brain. Therefore, extrapolating radiosurgery data derived from other tumor types is sub-optimal when treating patients with intracranial colorectal cancer metastases [10, 25]. We describe outcomes of radiosurgical treatment of brain metastasis from colorectal carcinoma at two tertiary care centers.

\section{Materials And Methods}

\section{Patients and methods}

We retrospectively reviewed data collected from 69 patients who received stereotactic radiosurgery to treat metastatic brain lesions originating from colorectal carcinoma. After excluding three patients who did not return to follow-up after radiosurgery, outcome regarding tumor progression and survival were available for 66 patients. Amongst these patients, 51 patients were treated at Miami Neuroscience center, Miami, FL (MNC) from 1993 to 2009, while 15 patients were treated at Johns Hopkins Hospital, Baltimore, MD (JHH) between 2003 and 2007. Approval was gained from the Institutional Review Board at JHH for chart review with waiver of consent and use of data from the MNC. The histopathological diagnosis was confirmed by biopsy of the tumor at primary site or from biopsy of a metastatic lesion at an extracranial site or from specimen obtained during craniotomy. According to our treatment protocol, neurological and radiological progression of metastatic disease was monitored with a follow-up initially at four weeks post-radiosurgery and subsequently at intervals of three months. Any local recurrence or appearance of new lesions was treated with either a subsequent session of radiosurgery or craniotomy.

\section{Gamma Knife}

All lesions were treated using a Leksell Gamma Knife model U, model B or model C (Elekta Instruments, Stockholm, Sweden). The recommendation for Gamma Knife therapy and the treatment plan were determined after consultation with a neurosurgeon and a radiation oncologist at the respective centers. Patients harboring multiple lesions were sometimes treated in multiple sessions. The prescription dose was based upon volume, number and location of lesions, with deep lesions and those located in proximity to critical neurovascular lesions receiving lower dose. The mean prescription dose at MNC was $16.93+2.66 \mathrm{~Gy}$, whereas patients at MTH received a similar dose with a median of $17.66+1.44 \mathrm{~Gy}$ (p-0.31).

\section{Statistical analysis}




\section{Cureus}

The demographic and clinical features of patients included in this study were summarized and compared between Mil and MNC. Frequencies and percentage were obtained when data was categorical and compared between two centers using Fisher's Exact test. Similarly, measures of variables continuous in nature were summarized as median and interquartile range (IQR) and compared using Wilcoxon Rank-Sum test. When treatment was administered in multiple sessions, we recorded as the treatment dose, the lowest dose received by lesions at all sessions. Survival times were calculated as time to mortality from the first radiosurgical treatment and also from initial diagnosis of brain metastasis. The progression-free survival (PFS) was calculated as time since treatment until subsequent intracranial progression or death. The median survival times and progression free survival were calculated using Kaplan Meier Analysis. Cumulative failure rates were derived from life table analysis. Patients were censored at last follow-up when there was no mortality or progression during Kaplan Meier analysis for survival and PFS, respectively. The median survival and PFS times were further stratified by the clinical and demographic features, such as treatment centers (JM and MNC), age groups (age < 65 years, age $>65$ years), Kamofsky performance scores (KPS) (KPS >80, $<70$ ), number of lesions (solitary lesion, two to three lesions, $>3$ lesions),volume (by quartiles) and interval between diagnosis of primary cancer to diagnosis of intracranial metastasis ( $<2$ year, $>2$ year). The survival times were compared between the strata and the two centers using Log Rank Test. Cox proportional hazard model was used to estimate relative risk of mortality attributable to covariates described initially using univariate analysis. We did not stratify our analysis by treatment center due to the small number of patients at each center. A stepwise backward selection of variables was undertaken for multivariate analysis. Cumulative incidence of brain metastases related (BMR) mortality was derived using non-brain metastases related (nonI3MR) mortality as competing risk factor and compared between covariates using Grays test.

All p-values reported are two-sided. The analysis was performed using Stata version 12 (Stata Corp LP: College Station, TX) and R version 2.13.0 (2011-04-13).

\section{Results}

The clinical and demographic characteristics of the patients at two centers have been summarized in Table 1. Mean age of patients in this series was 65.10+11.91 years. Median KPS of the patient population was 80 with $72 \%$ patients having KPS $>70$. The majority of patients in this series had colon cancer (64 (92.75\%) patients), while rectal carcinoma was present in 5 (7.25\%) patients. Median time to development of brain metastasis from diagnosis of the primary cancer was 31.95 months (IQR: $15.03-50.93$ months). Three patients had synchronous brain metastasis at the time of diagnosis of colorectal cancer; follow-up was available for two of these patients: one patient died at 1.45 and the other at 4.31 months after radiosurgical treatment.

\begin{tabular}{|c|c|c|c|}
\hline & MNC & JHH & p \\
\hline $\mathbf{N}$ & 54 & 15 & \\
\hline \multicolumn{4}{|l|}{ Age } \\
\hline Age & $66.5+11.83$ & $60.0+12$ & 0.06 \\
\hline Age $>65$ & $32(59.26)$ & 5 (33.33) & \multirow{2}{*}{0.08} \\
\hline Age $<65$ & 22 (40.74) & 10 (66.67) & \\
\hline \multicolumn{4}{|l|}{ Gender } \\
\hline Males & 30 (55.56) & 11 (73.33) & \\
\hline
\end{tabular}




\section{Cureus}

Female

24 (44.44)

Number of lesions

1 lesions

2-3 lesions

$>3$ lesions

Lesion volume

$<5.6 \mathrm{ml}(\mathrm{p} 25)$

5.6-11.4ml (p25-p50)

11.4-17.3ml (p50-p75)

$>17.3 \mathrm{ml}(>\mathrm{p} 75)$

KPS

Median KPS (IQR)

KPS $\leq 70$

KPS $>70$

Time to diagnosis of metastasis

$\leq 12$ months

$>12$ months

Treatment

GK

GK+WBRT

Median treatment dose (Gy)
7 (15.56)

38 (84.44)

$28(51.85)$

$10(66.67)$

18 (33.33)

3 (20.0)

8 (14.81)

2 (13.33)

9 (16.67)

$10(66.67)$

12 (24.07)

3 (20)

16 (29.63)

1 (6.67)

16 (29.63)

2 (13.33)

80 (70-90)

90 (80-100)

$13(32.50)$

1 (10)

$41(67.50)$

9 (90)

$46(88.46)$

6 (11.54)

$17(16-18)$
13 (86.67)

2 (13.33)

1 (6.67)

14 (93.33)

0.57

$18(16-18)$
0.61

0.11

0.35

0.17

TABLE 1: Demographics and Clinical Features of Patients

At least one follow-up visit was available for 66 patients, who were included in the study. Fiftyeight (84.06\%) patients died over a mean follow-up of 7.03+12.08 months after radiosurgery. Median survival from time of radiosurgery was 4.05 months (95\% CI: 2.40-5.36 months) and from the time of diagnosis of brain metastasis was 6.67 months (95\% CI: 5.06-10.52 months) observed during the study period. A comparable survival (p-0.21) was observed at two centers 3.91 (95\% CI: 2.2-4.86 months) at MNC and 7.07 (95\% CI: 1.67-15.36 months) at Hifi. Survival at six months and one year was 34.77\% (95\% CI: 23.18-46.62\%) and 14.64\% (95\% CI: 6.95-

$25.04 \%)$. Data regarding cause of death was available for 45 patients, of whom three (6.67\%) had brain metastasis related mortality and 42 (93.33\%) patients died from extracranial disease progression. Three patients who died from brain metastasis related causes survived for 1.88 , 2.43 and 18.42 months since radiosurgery. 


\section{Cureus}

Data regarding KPS scores at time of Gamma Knife were available for 47 patients. Amongst 33 patients with KPS > 70, median survival was 6.91 months, and 27 (81.8\%) patients died in the time period captured by this study. Median survival in 14 patients with KPS <70 was 1.74 months (p-0.004) with 100\% mortality during follow-up (Figure 1). There was no significant difference in survival by factors, such as number of lesions, total volume of lesion, or age of the patient (Table 2, Figures 1-4).

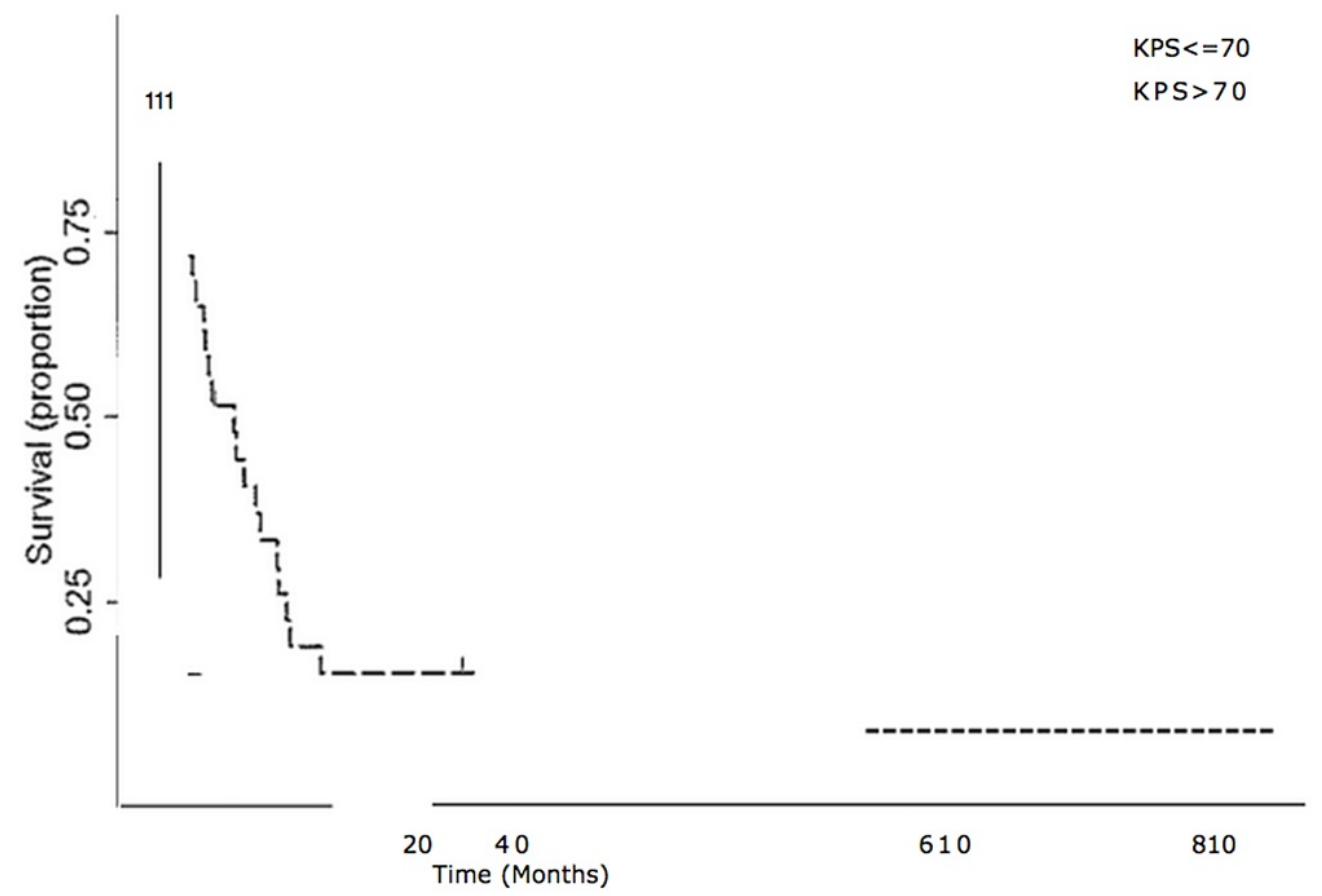

FIGURE 1: Survival by KPS

\section{Strata}

Center

MNC

Hopkins

Age

Age $<65$

Age $\geq 65$
N Dead (\%)
Median Survival

(months)

$3.91(2.2-4.84)$

7.07 (1.6 - 15.36)
48

(94.11)

$15 \quad 10$

(66.67)

\section{Log-rank p-value (between} strata)

0.21

29

23

(83.18)

$4.77(2.36-6.91)$

0.87

Gender

$37 \quad 35$

$(89.74)$ 


\section{Cureus}

$\begin{array}{lllll}\text { Females } & 27 & (83.18) & 4.11(2.43-6.91) & 0.87 \\ \text { Male } & 39 \begin{array}{l}35 \\ (89.74)\end{array} & 4.05(2.00-7.07)\end{array}$

Number of lesions

\begin{tabular}{|c|c|c|c|}
\hline 1 lesions & 35 & $\begin{array}{l}29 \\
(82.85)\end{array}$ & $3.95(2.04-7.08)$ \\
\hline 2-3 lesions & 21 & $19(90.4)$ & $3.16(1.58-5.43)$ \\
\hline$>3$ lesions & 10 & $10(100)$ & $5.1(1.78-9.93)$ \\
\hline \multicolumn{4}{|l|}{ Lesion volume } \\
\hline$<5.6 \mathrm{ml}(\mathrm{p} 25)$ & 19 & $\begin{array}{l}15 \\
(78.94)\end{array}$ & $6.91(1.48-9.93)$ \\
\hline $5.6-11.4 \mathrm{ml}$ (p25-p50) & 15 & $\begin{array}{l}14 \\
(93.33)\end{array}$ & $3.03(1.88-7.07)$ \\
\hline $11.4-17.3 \mathrm{ml}(\mathrm{p} 50-\mathrm{p} 75)$ & 15 & $\begin{array}{l}14 \\
(93.33)\end{array}$ & $4.74(2.2-4.9)$ \\
\hline$>17.3 \mathrm{ml}(>\mathrm{p} 75)$ & 17 & $\begin{array}{l}15 \\
(88.23)\end{array}$ & $3.16(1.51-7.6)$ \\
\hline
\end{tabular}

Distribution of volume

Solitary small lesion (volume

$<5.6 c c$ )

$9 \quad 6(66.7) \quad 8.48(0.95-$. $)$

0.037

2-3 lesions with total volume

$<5.6 \mathrm{cc}$

$65(83.33) \quad 1.48(0.85-$ - $)$

$>3$ lesions with total volume $<5.6 \mathrm{cc}$

$4 \quad 4(100) \quad 5.36(1.78-$.

Solitary large lesion (volume

2623

$>5.6 \mathrm{cc}$ )

$2.43(2.04-4.9)$

0.62

2-3 lesions with total volume

$>5.6 \mathrm{cc}$

$15 \quad 14$

$4.31(1.88-10.1)$

$>3$ lesions with total volume $>5.6 \mathrm{cc}$

$6 \quad 6(100)$

$4.84(1.91-$.

KPS

$\mathrm{KPS} \leq 70$

$1414(100)$

$1.74(1.12-2.43)$

0.0004

KPS $>70$

$33^{27}$

(88.18)

$4.11(2.2-7.6)$

Time to diagnosis of metastasis

$\leq 12$ months

$6 \quad 6(100) \quad 3.95(1.45-$.

0.24

$>12$ months

5143

(84.33)

$4.11(2.2-7.6)$ 


\section{Cureus}

Treatment

GK

$57 \quad 51$

$(89.47)$

$4.05(2.65-5.43)$

0.75

GK + WBRT

$8 \quad 6(75) \quad 4.31(1.12-$.)

Dose

>18Gy

$43 \quad 39$

$(90.69)$

$2.8(1.88-4.11)$

0.66

$\leq 18 \mathrm{~Gy}$

$23 \begin{array}{ll}19 \\ (82.60)\end{array}$

$9.21(4.05-10.63)$

TABLE 2: Survival Time from Gamma Knife Procedure

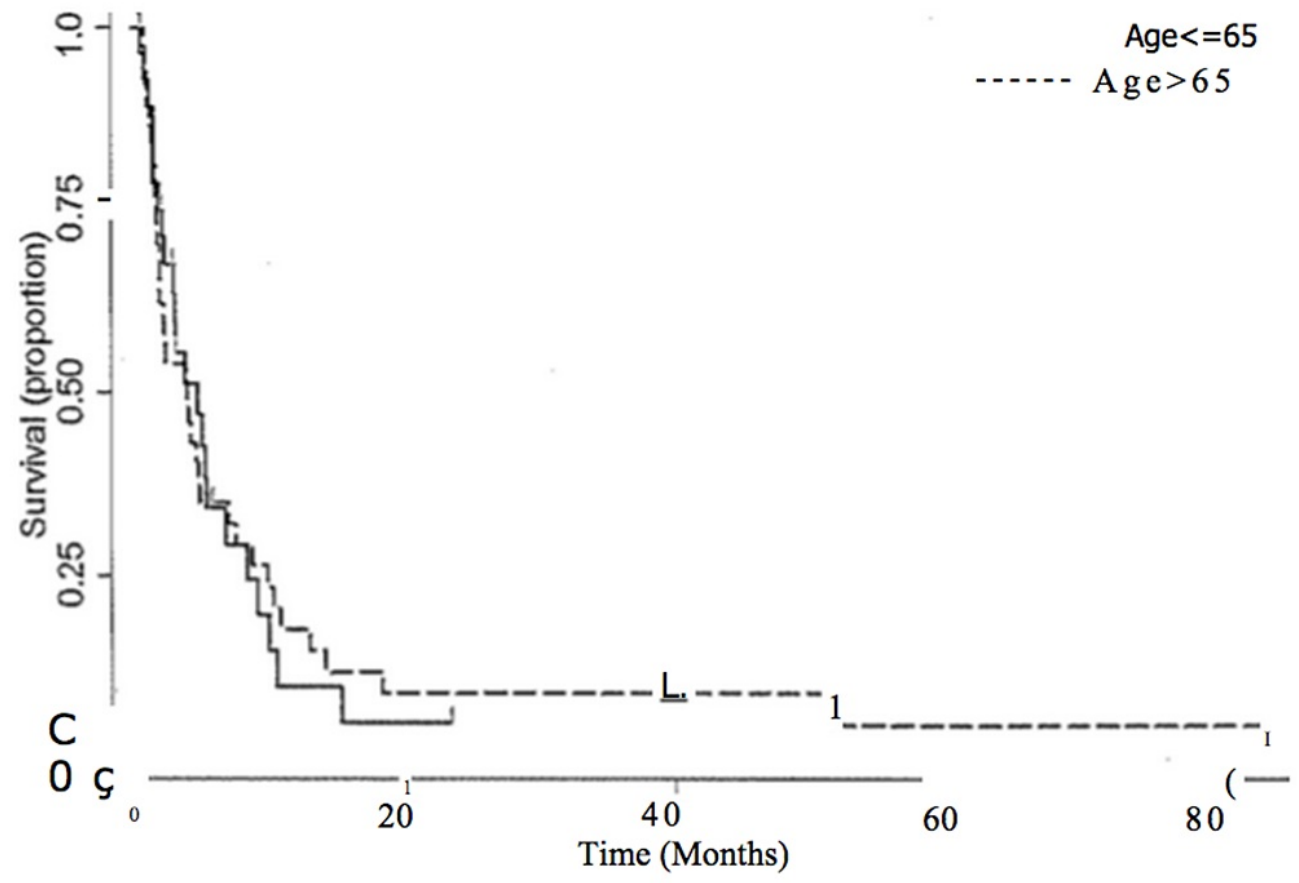

FIGURE 2: Survival by Age 


\section{Cureus}

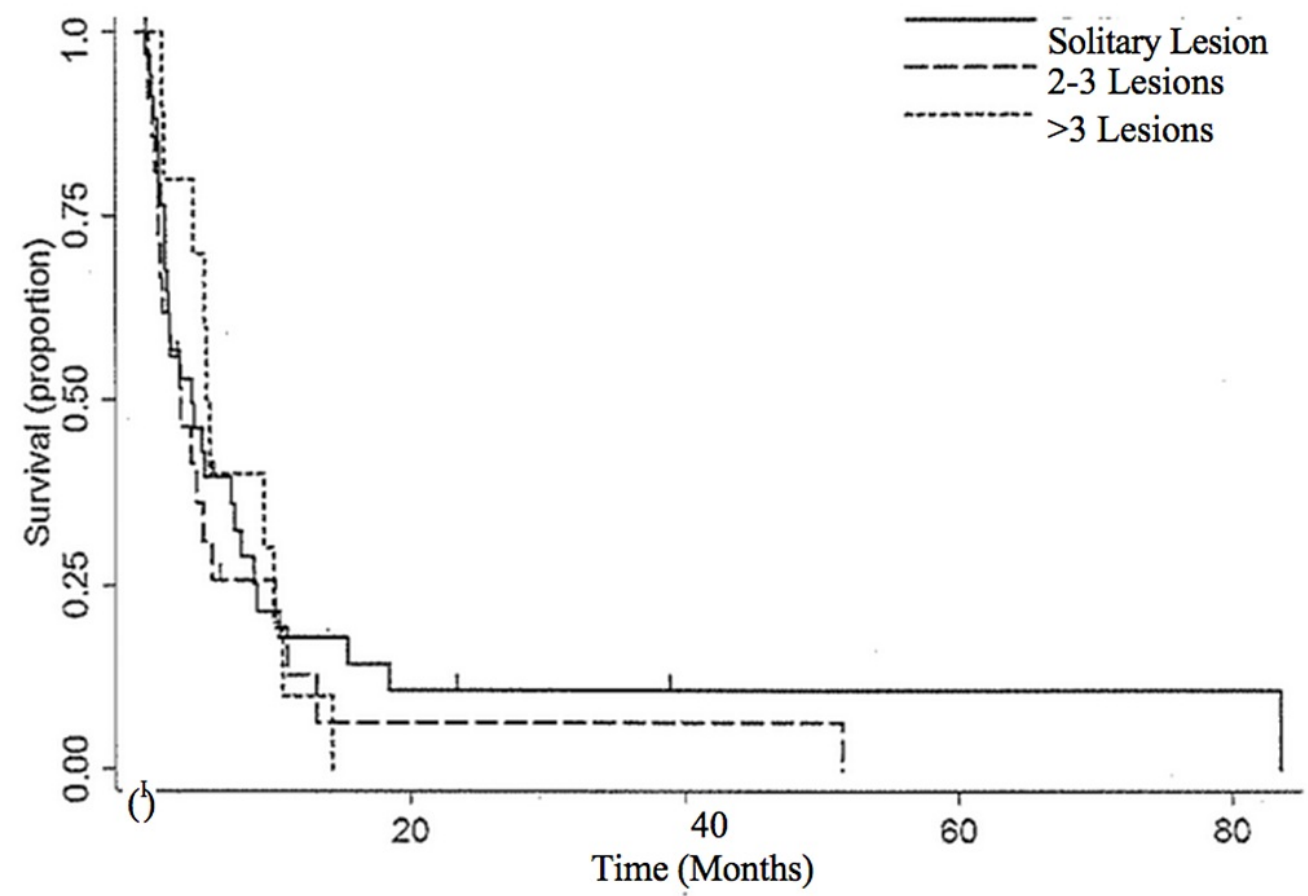

FIGURE 3: Survival by Lesion Number

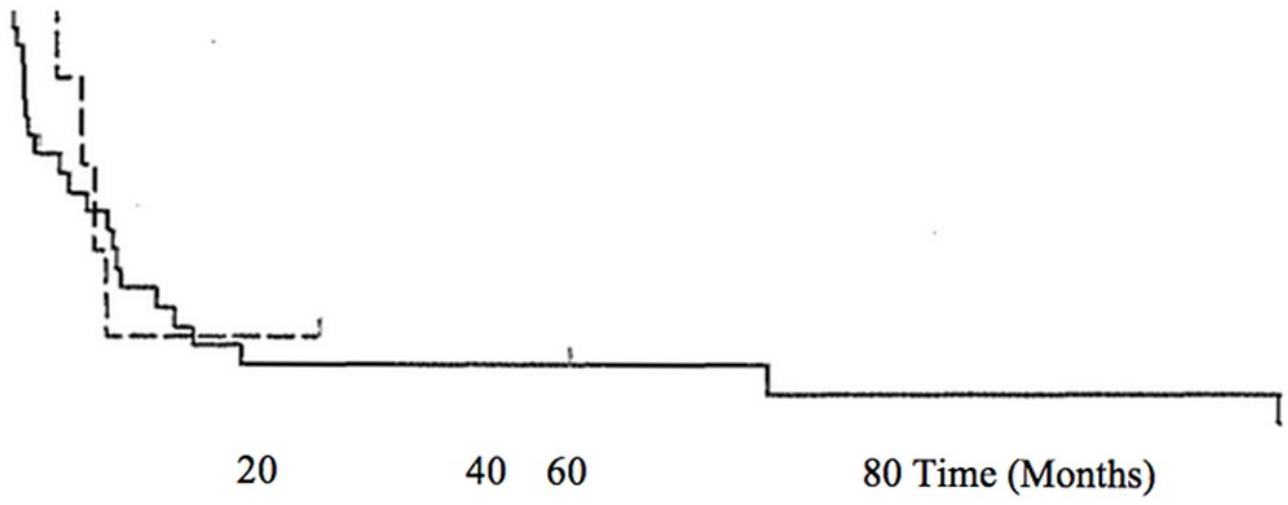

FIGURE 4: Survival by Total Volume 


\section{Cureus}

\section{Prognostic factor}

Age $\geq 65$

$\mathrm{KPS} \leq 70$

Lesions $>3$

Volume $>8.4 \mathrm{cc}$

Synchronicity of brain metastasis

Dose $<18$

Male

GK+WBRT
Odds Ratio $(95 \% \mathrm{Cl})$

$0.95(0.55-1.63)$

$3.22(1.62-6.39)$

$0.90(0.45-1.73)$

$1.45(0.82-2.56)$

$1.66(0.69-4.01)$

$2.69(1.49-4.84)$

$1.05(0.62-1.80)$

1.14(0.48-2.72)
0.25

0.001

0.21

0.87

0.001

0.77

0.19

0.75

\section{TABLE 3: Univariate Hazard Ratios}

When evaluating treatment related factors, higher dose of radiation was associated with better survival (Figure 5). Patients who were treated with a dose less than 18 Gy survived for a median of 2.8 months $(\mathrm{N}:=43$, mortality: $90.69 \%)$ as compared to 9.21 months in patients $(\mathrm{N}=23$, mortality: $82.60 \%$ ) who were treated with a dose of $18 \mathrm{~Gy}$ or more ( $\mathrm{p}=0.0012)$. Furthermore, patients who received a repeat Gamma Knife treatment for recurrence of metastatic lesions survived for significantly longer duration (survival: 10.36 months; mortality: $92.85 \%$ ) than patients who did not receive the subsequent procedure (survival: 3.03 months; mortality: 86.53\%), $\mathrm{p}=0.0079$.

\section{Dose $<=18$}

Dose>1 8 


\section{Cumulative incidence of mortality caused by brain metastases and other causes}

Cumulative incidence of brain metastasis related (BMR) and non-BMR mortality using competing risk analysis at 12 months was 4.32\% (95\% Cl: $0.7-13.19 \%)$ and $84.16 \%$ (95\%Cl 68.72-92.36\%), respectively. While, at 24 months, cumulative incidence of BMR and non-BMR mortality was 6.63\% (95\%0: 1.5-17.32\%) and $88.76 \%$ (95\% Cl $73.76-95.43 \%)$, respectively.

On univariate analysis, KPS $<70$ and no repeat treatment, despite recurrence, were found to be significant predictors of mortality. No other variables, including number of lesions, volume of lesions, adjuvant radiotherapy, age, treatment center, diagnosis of intracranial metastasis within two years of diagnosis of primary colorectal cancers or dose was found to be significant predictor of mortality. Furthermore, when all of these variables were included in a multivariate analysis, only KPS $>70$ was an independent predictor of survival with patients having KPS $>70$ being $69 \%$ less likely to die. However, when only variables with $\mathrm{p}$ value $<0.1$ were included in multivariate analysis, both KPS $>70$ and repeat radiosurgery for recurrence were found to be independent predictors of survival. This was so due to greater number of patients with complete data for these two variables when compared to all the variables included in the initial model. On this multivariate analysis, patients with KPS $>70$ were $27.31 \%$ less likely to die than patients with lower KPS (Hazard Ratio: 0.2731, 95\% CI: 0.13 -0.56, p=0.001). Also, patients who had subsequent radiosurgery for local recurrence or relapse of intracranial metastasis were 36\% less likely to die than patients not treated (Hazard Ratio: 0.3619, 95\%0: 0.15-0.82, $\mathrm{p}=0.016$ ).

\section{Intracranial progression-free survival}

Median intracranial progression free survival (PFS) was 3.03 months in this series with 20.1\% patients diagnosed with progression prior to mortality. Progression was treated with a repeat session of radiosurgery. The median PFS in patients with KPS $<70$ was 1.74 months as compared to 6.91 months in patients with higher KPS ( $\mathrm{p}=0.004)$. Importantly, the PFS did not differ by number of lesions or the total volume of lesions.

\section{Discussion}

This series describes radiosurgical outcomes of patients with colorectal carcinoma metastases to the brain. An overall median survival of 4.05 months was observed from the time of radiosurgery and 6.67 months after diagnosis of brain metastasis. This contrasts with patients harboring brain metastasis from breast cancer where a median survival of 11-13 months from radiosurgery has been reported [15, 19, 20, 27]. Similarly, higher survival [11-13] is also observed after radiosurgery in lung cancer patients with brain metastases [I5-16]. Our results are in concordance with the three existing series studying colorectal cancer, which report survival ranging from 5-6.7 months after radiosurgery [3,25-26].

\section{Extracranial control, Karnofsky performance, status and age}

Variables, such as control of the primary lesion, age and KPS, have been widely studied in the radiosurgery literature [14-16, 20, 24,28-29] and these form the key components of the commonly used RPA scales $[13,30]$. In this study, Karnofsky performance score (KPS)>70 was associated with higher survival on both univariate and multivariate analysis. Several studies have found the association of KPS $>70$ with a favorable survival in colorectal carcinoma [3,14,25] 
as well as other histologies [13,15-17,20,27-29]. In the current study, patients with KPS<70 had a median survival of 1.74 months when compared to 6.91 months in patients who had a higher KPS scores. This finding corroborates the practice that patients with KPS $<70$ should be offered palliative treatment with steroids or whole brain radiation, since radiosurgical or surgical intervention does not improve survival.

Patients with metastases to the brain from colon cancer are rarely free of extracranial disease [26]. As a result, some groups have found that increasing extracranial disease burden is associated with mortality in patients with metastatic colorectal cancer to the brain $[3,10,14,25$ 26]. It is thought that extracranial dissemination of colorectal cancer often precedes metastasis to the brain. This could be a result of hematogenous spread of colorectal cancer to the liver via portal drainage. Liver metastases are frequent, occurring in up to $79 \%$ of colon cancer patients and carries a poor prognosis [10, 31]. However, in this study, systemic tumor burden was not associated with survival. Although much of the existing opinion and literature considers extracranial control of primary disease to be critical for survival, a few surgical and radiosurgical series of colorectal carcinoma have not found a strong role for systemic disease burden on survival.

In a large surgical series of 100 patients reported by Hammoud, et al., the presence of liver or lung metastatic lesions from colorectal carcinoma was associated with poor survival only on univariate analysis, but this association did hold on multivariate analysis. Surgical resection of brain metastasis was the only significant variable that impacted survival with median survival of nine months in those who underwent surgical resection of lesions as compared to three months in radiotherapy treated patients. Similar to surgical series, some radiosurgical series fail to show statistically proven association of survival and long-term remission with extracranial control. Hasegawa, et al., reported an incidence of $30.8 \%$ lung metastasis and $17.9 \%$ liver metastasis [25]. Patients with liver metastasis survived for four months as compared to eight months in those without liver metastasis, but this trend did not attain statistical significance $(\mathrm{p}=0.50)$. Additionally, patients with well-controlled systemic tumor burden likely have widespread micrometastatic disease that is difficult to pulmonary metastasis survived for a median of eight months as compared to five months in those with no lung lesions, and this too was not statistically significant $(\mathrm{p}=0.65)$. However, in view of these trends and other existing literature, they attributed poor survival in patients with colorectal cancer to uncontrolled extracranial metastasis, particularly to the liver [25]. Similarly, in a series reported by De Silva, et al., higher survival was associated with absence of extracranial lesions (7.3 versus 5.1 months) but like others described above, this association did not reach statistical significance $(p=0.86)$. Scoeggl and colleagues also reported a median of 3.5 months survival when extracranial dissemination of colorectal cancer was present as compared to 5.9 months when extracranial disease was controlled $(\mathrm{p}=0.25)$. One potential reason for the lack of association is that even those patients who appear to have well-controlled systemic tumor burden likely have widespread micrometastatic disease that is difficult to detect but impacts survival. Though our series is the largest in the radiosurgical literature reported thus far, it does not investigate the role of extracranial control in management of colorectal cancer brain metastasis due to lack of these data.

\section{Tumor volume and lesion number:}

In this series, no significant association was found between the number of lesions or tumor volume and the median time to mortality (Figures 6, 7). Patients with solitary metastasis survived for a median of 3.95 months (95\% CI: 2.04-7.07 months) as compared to 4.31 months (95\% CI: $1.91-5.43$ months) in patients with more than one lesion ( $\mathrm{p}=0-0.77)$. Univariate Cox regression also failed to find the number of lesions as a predictor of survival. We arbitrarily classified the lowest quartiles of volume $(<=5.6 \mathrm{cc})$ as small lesions and explored the influence of distribution of total volume on survival. Patients with small lesions in this series survived for a 


\section{Cureus}

median of 6.91 months (95\%CI 1.48- 9.93 months), while those with larger lesions survived for 3.95 (95\%CI 2.36-4.84), ( $\mathrm{p}=0.298)$. While our results are largely in agreement with other radiosurgery series for colorectal carcinoma brain metastasis that did not find volume or number of lesions to predict survival [3,25-26], in our series, solitary lesions less than $5.6 \mathrm{cc}$ in volume had a significantly higher survival ( $\mathrm{N}=9$, median survival: 8.48 months, 95\% CI: 0.95.mortality $67 \%$ ) than patients with greater volume or multiple lesions ( $\mathrm{N}=57$, Median survival: 3.91 months, 95\% CI: 3.03-5.43 months, mortality: 91.22\%) $\mathrm{P}=0.025$.

Solitary lesion \& Vol $<=5.6 \mathrm{cc}$ 2-3 lesions \& Vol< $<=5.6 \mathrm{cc}>3$

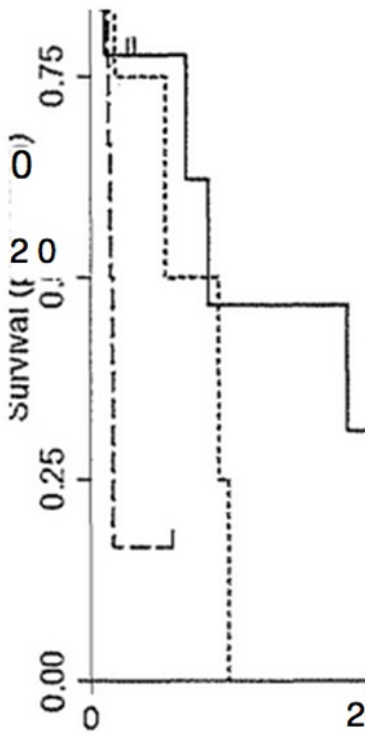
lesions \& Vol $<=5.6 \mathrm{cc}$

FIGURE 6: Survival by Lesion Number and Volume $<5.6 \mathrm{cc}$ 


\section{Cureus}

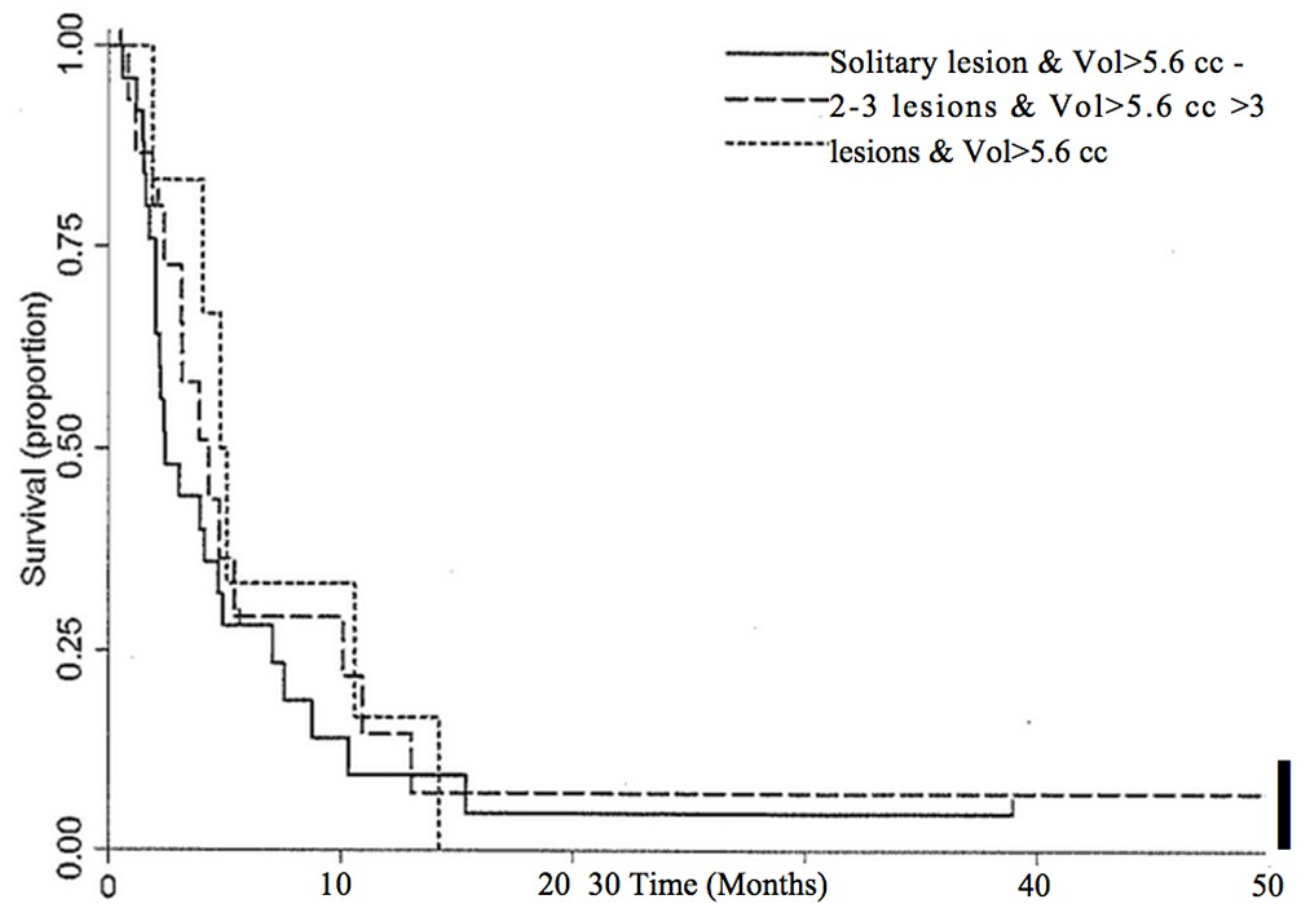

FIGURE 7: Survival by Lesion Number and Volume $>5.6 \mathrm{cc}$

Furthermore, when total volume did not exceed $5.6 \mathrm{cc}$, we found significantly lower survival with increasing number of lesions; however, this association was not true for higher total lesion volume. (This interaction of volume and lesion numbers did not stand after multivariate analysis, possibly due to lack of power). This compares favorably with other histological studies whose results suggest patients with solitary or at least fewer than three lesions or smaller total tumor volume have longer overall and progression free survival [29,32-33]. Similarly, Diluna, et al. reported higher survival in patients with fewer than four lesions and total volume less than $5 \mathrm{cc}$ [34]. Other series have proposed intracranial location to be possible determinants of mortality, although no statistical association was established [10]. However, elucidating the role of volume and number of lesions in determining mortality, particularly in the context of lesions in deep or eloquent sites, is challenging since colorectal carcinoma brain metastases are infrequent and most studies include small samples size. Given the limited available data, it is unclear whether colon cancer constitutes a unique histological class where survival is not linked to number or volume of intracranial lesions or if those factors do play a key role in determining outcome. As a result, further well-controlled prospective studies are needed to determine if the guidelines used for other histologies apply to this subgroup of patients.

\section{Adjuvant radiotherapy}

This series (Figure 8 ) found no additional survival benefit for patients who received adjuvant whole brain radiotherapy. Survival in the radiosurgery only group was 4.05 months versus 4.31 months in the adjuvant radiotherapy group (v0.75). Furthermore, progression free survival (PFS) did not increase in patients who received adjuvant radiotherapy (median PFS 3.03 months) as compared to those who received only radiosurgery (median PFS 3.03 months) $(\mathrm{p}=0.57)$. Other series have similarly found no improvement in overall survival or progression free survival $[3,14,25]$. Sperduto and colleagues, in a multi-institutional study, found a median survival 7.13 months with adjuvant radiotherapy as compared to 7.33 months with radiosurgery alone ( $\mathrm{p}=0.45$ ). Likewise, Da Silva, et al. reported 6.9 months of median survival with adjuvant 
radiotherapy, whereas patients who received only radiosurgery survived for a median of 6.6 months ( $p=0.93$ ). A team led by Hasegawa reported a median survival of nine months with adjuvant radiotherapy as compared to five months without ( $p=0.81)$ [25]. However, in this series, prior surgery was correlated with better survival (11 months versus five months, $\mathrm{p}$ 0.047 ) [25]. Similar to this Hammoud, et al. reported decline in risk of mortality by $87 \%$ and $88 \%$, respectively, in groups treated with surgical resection $(\mathrm{p}<0.001)$ and surgery with adjuvant radiotherapy $(\mathrm{p}<0.001)$ as compared to conservative management. Our series did not address the role of surgery due to lack of relevant data.

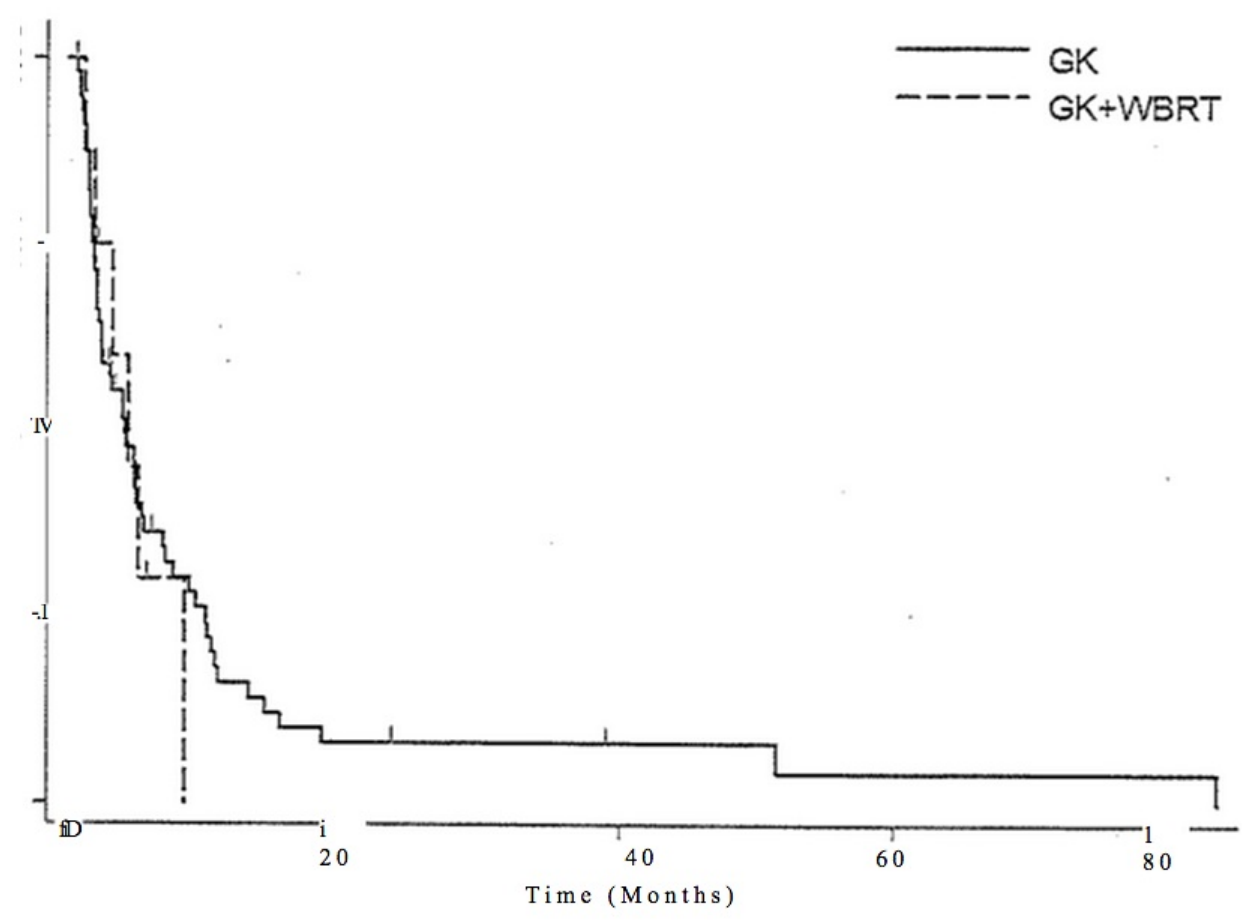

FIGURE 8: Survival by Adjuvant

\section{Multiple procedures for recurrence:}

In this study, patients who received additional treatments for progression had a greater survival than patients who received radiosurgery for only the initial metastatic lesions (median survival: 10.36 months 95\% CI: 4.77 - 14.24 months versus median survival: 3.03 months, 95\%CI 1.914.31 months $\mathrm{p}=0.0079)$. On univariate analysis, patients who received additional procedures were $59 \%$ less likely to die when compared to those with only a single procedure (Hazard ration: 0.411; 95\% CI 0.209-0.807, p-0.010). Multivariate analysis revealed administration of additional Gamma Knife sessions to be an independent predictor of survival (Hazard ratio: 0.361, 95\% CI: $0.157-0.829, \mathrm{p}-0.016)$. In this series, 14 (20.29\%) patients underwent repeat radiosurgery for recurrence and may represent a subgroup of patients with relatively less aggressive cancer or may have different patterns of systemic spread, thereby confounding the results.

\section{Conclusions}

Colorectal cancer metastatic to the brain carries with it a poor prognosis. A moderate improvement in survival after radiosurgery is seen only in the subgroup of patients with KPS $>70$. Larger prospective studies are needed to identify ideal candidates for aggressive management. 


\section{Additional Information \\ Disclosures}

Human subjects: Consent was obtained by all participants in this study. The Institutional Review Board at Johns Hopkins Hospital issued approval N/A. Animal subjects: All authors have confirmed that this study did not involve animal subjects or tissue. Conflicts of interest: In compliance with the ICMJE uniform disclosure form, all authors declare the following: Payment/services info: All authors have declared that no financial support was received from any organization for the submitted work. Financial relationships: All authors have declared that they have no financial relationships at present or within the previous three years with any organizations that might have an interest in the submitted work. Other relationships: All authors have declared that there are no other relationships or activities that could appear to have influenced the submitted work.

\section{References}

1. Boyle P, Langman JS: ABC of colorectal cancer: Epidemiology. BMJ. 2000, 321:805-808.

2. Flickinger J, Kondziolka D, Lunsford L, et al: A multi-institutional experience with stereotactic radiosurgery for solitary brain metastasis. Int J Radiat Oncol Biol Phys. 1994, 28:797-802.

3. Da Silva AN, Nagayama K, Schlesinger DJ, Sheehan JP: Gamma Knife surgery for brain metastases from gastrointestinal cancer. J Neurosurg. 2009, 111:423-430. 10.3171/2008.9.JNS08281

4. Barnholtz-Sloan JS, Sloan AE, Davis FG, Vigneau FD, Lai P, Sawaya RE: Incidence proportions of brain metastases in patients diagnosed (1973 to 2001) in the Metropolitan Detroit Cancer Surveillance System. J Clin Oncol. 2004, 22:2865-2872.

5. Delattre JY, Krol G, Thaler HT, Posner JB: Distribution of brain metastases . Arch Neurol. 1988, 45:741-744.

6. Nieder C, Pawinski A, Balteskard L: Colorectal cancer metastatic to the brain: time trends in presentation and outcome. Oncology. 2009, 76:369-374. 10.1159/000210026

7. Sundermeyer ML, Meropol NJ, Rogatko A, Wang H, Cohen SJ: Changing patterns of bone and brain metastases in patients with colorectal cancer. Clin Colorectal Cancer. 2005, 5:108-113.

8. Ko FC, Liu JM, Chen WS, Chiang JK, Lin TC, Lin JK: Risk and patterns of brain metastases in colorectal cancer: 27-year experience. Dis Colon Rectum. 1999, 42:1467-1471.

9. Patchell R, Tibbs P. Regine W, et al: Postoperative radiotherapy in the treatment of single metastases to the brain: a randomized trial. JAMA. 1998, 280:1485-1489.

10. Hammoud MA, McCutcheon IE, Elsouki R, Schoppa D, Patt YZ: Colorectal carcinoma and brain metastasis: distribution, treatment, and survival. Ann Surg Oncol. 1996, 3:453-463.

11. Tosoni A, Lumachi F, Brandes AA: Treatment of brain metastases in uncommon tumors . Expert Rev Anticancer Ther. 2004, 4:783-793.

12. Alexander Er, Moriarty T, Davis R, et al: Stereotactic radiosurgery for the definitive, noninvasive treatment of brain metastases. J Natl Cancer Inst. 1995, 87:34-40.

13. Gaspar L, Scott C, Rotman M, Asbell S, Phillips T, Wasserman T, McKenna WG, Byhardt R: Recursive partitioning analysis (RPA) of prognostic factors in three Radiation Therapy Oncology Group (RTOG) brain metastases trials. Int J Radiat Oncol Biol Phys. 1997, 37:745751.

14. Sperduto P, Chao S, Sneed P, et al: Diagnosis-Specific Prognostic Factors, Indexes, and Treatment Outcomes for Patients with Newly Diagnosed Brain Metastases: A Multiinstitutional Analysis of 4,259 Patients. Inti Radiat Oncol Biol Phys. 2010, 77:655-661. 10.1016/j.ijrobp.2009.08.025.

15. Frazier JL, Batra 5, Kapoor S, et al: Stereotactic radiosurgery in the management of brain metastases: an institutional retrospective analysis of survival. Int J Radiat Oncol Biol Phys. 2010, 76:1486-1492. 10.1016/j.ijrobp.2009.03.028

16. Sheehan JP, Sun MH, Kondziolka D, Flickinger J, Lunsford LD: Radiosurgery for non-small cell lung carcinoma metastatic to the brain: long-term outcomes and prognostic factors influencing patient survival time and local tumor control. J Neurosurg. 2002, 97:1276-1281. 
17. Flannery T, Suntharalingam M, Kwok V, et al: Gamma knife stereotactic radiosurgery for synchronous versus metachronous solitary brain metastases from non-small cell lung cancer. Lung Cancer. 2003, 42:327-333.

18. Flannery $\mathrm{T}$, Suntharalingam M, Regine W, et al: Long-term survival in patients with synchronous, solitary brain metastasis from non-small-cell lung cancer treated with radiosurgery. Int J Radiat Oncol Biol Phys. 2008, 72:19-23. 10.1016/j.ijrobp.2007.12.031

19. Firlik KS, Kondziolka D, Flickinger JC, Lunsford LD: Stereotactic radiosurgery for brain metastases from breast cancer. Ann Surg Oncol. 2000, 7:333-338.

20. Kased N, Binder D, McDermott M, et al: Gamma Knife radiosurgery for brain metastases from primary breast cancer. Int J Radiat Oncol Biol Phys. 2009, 75:1132-1140. 10.1016/j.ijrobp.2008.12.031

21. Goyal A, Singh AK, Gupta V, Tatke M: Spinal epidural cavernous haemangioma: a case report and review of literature. Spinal Cord. 2002, 40:200-202.

22. Selek U, Chang E, Hassenbusch Sr, et al: Stereotactic radiosurgical treatment in 103 patients for 153 cerebral melanoma metastases. Int J Radiat Oncol Biol Phys. 2004, 59:1097-1106.

23. Mori Y, Kondziolka D, Flickinger JC, Logan T, Lunsford LD: Stereotactic radiosurgery for brain metastasis from renal cell carcinoma. Cancer. 1998, 83:344-353.

24. Powell J, Chung C, Shah H, et al: Gamma Knife surgery in the management of radioresistant brain metastases in high-risk patients with melanoma, renal cell carcinoma, and sarcoma. J Neurosurg. 2008, 109:122-128. 10.3171/JNS/2008/109/12/S19

25. Hasegawa T, Kondziolka D, Flickinger JC, Lunsford LD: Stereotactic radiosurgery for brain metastases from gastrointestinal tract cancer. Surg Neurol. 2003, 60:506-514.

26. Schoeggl A, Kitz K, Reddy M, et al: Stereotactic radiosurgery for brain metastases from colorectal cancer. Int J Colorectal Dis. 2002, 17:150-155.

27. Goyal S, Prasad D, Harrell F Jr, Matsumoto J, Rich T, Steiner L: Gamma knife surgery for the treatment of intracranial metastases from breast cancer. J Neurosurg. 2005, 103:218-223.

28. Sneed P, Suh J, Goetsch 5, et al: A multi-institutional review of radiosurgery alone vs. radiosurgery with whole brain radiotherapy as the initial management of brain metastases. Int J Radiat Oncol Biol Phys. 2002, 53:519-526.

29. Petrovich Z, Yu C, Giannotta SL, O'Day S, Apuzzo ML: Survival and pattern of failure in brain metastasis treated with stereotactic gamma knife radiosurgery. J Neurosurg. 2002, 97:499-506.

30. Gaspar LE, Scott C, Murray K, Curran W: Validation of the RTOG recursive partitioning analysis (RPA) classification for brain metastases. Int J Radiat Oncol Biol Phys. 2000, 47:10011006.

31. Dawson L, Russell A, Tong D, et al: Adenocarcinoma of the sigmoid colon: sites of initial dissemination and clinical patterns of recurrence following surgery alone. J Surg Oncol. 1983, 22:95-99.

32. Bhatnagar AK, Flickinger JC, Kondziolka D, Lunsford LD: Stereotactic radiosurgery for four or more intracranial metastases. Int J Radiat Oncol Biol Phys. 2006, 64:898-903.

33. Jawahar A, Shaya M, Campbell P, Ampil F, Willis BK, Smith D, Nanda A: Role of stereotactic radiosurgery as a primary treatment option in the management of newly diagnosed multiple (3-6) intracranial metastases. Surg Neurol. 2005, 64:207-212.

34. DiLuna ML, King JT Jr, Knisely JP, Chiang VL: Prognostic factors for survival after stereotactic radiosurgery vary with the number of cerebral metastases. Cancer. 2007, 109:135-145. 